\title{
Accessibility patterns: West Mediterranean Case Study ${ }^{1}$
}

\author{
Rafael Rodrigo, Nati Franco, Oriol Biosca, Andreu Ulied \\ MCRIT \\ Salvador Espriu 83, 08005 Barcelona, Spain \\ rodrigo@mcrit.com,nati@mcrit.com, obiosca@mcrit.com, ulied@mcrit.com
}

\begin{abstract}
The accessibility patterns in the West Mediterranean region follow the geographic constraints of the region closely. Being a narrow coastal corridor for most of the territory, most of the population and activities are concentrated along the sea shore. The backcountry is substantially less populated, especially in mountain areas, and infrastructure endowment is more limited, yielding poorer accessibility patterns in comparison. The case of the Balearic Islands shows the effect of insularity on accessibility patterns. The influence of the Barcelona agglomeration reaches approximately all NUTS-3 capitals in Catalonia, and in some cases even Perpignan in France. The metropolitan agglomerations of Valencia and Alacant tend to merge with each other in most of the accessibility indicators, despite the fact that mountainous topography in the areas in between both agglomerations provides locally some areas of low accessibility. The new high speed rail lines planned in the region increase the opportunities of medium cities located in between large agglomerations as they are in the position to offer high live standards while allowing for daily commuting onto large labour markets, yet now only on paper. All over the region, accessibility of public services is more homogenous than accessibility of the population and jobs as public services are relatively decentralized.
\end{abstract}

Keywords: accessibility, transport infrastructure, accessibility pattern, TEN-T, GIS, West Mediterranean.

\section{Case study introduction}

The West Mediterranean Regions case study covers Spanish and French cross border regions, and Andorra. It stretches over three different states (NUTS 0), covering three Spanish autonomous communities (NUTS 2) and a part of a French region, 10 different NUTS 3 areas, several hundred counties, and about 2,000 municipalities (LAU2). It covers more than 1,000 km of coastal areas, mountain regions with highs up to $3,000 \mathrm{~m}$, four islands, highly populated metropolitan regions

1 This paper has been developed under the applied research project TRansport ACCessibility at regional/local scale and patterns in Europe led by Spiekermann \& Wegener Urban and Regional Research (S\&W) Germany. It has been financed by the ESPON 2013 Programme and its financial support is gratefully acknowledged. Texts, maps and conclusions stemming from research projects under the ESPON programme presented in this paper do not necessarily reflect the opinions of the ESPON Monitoring Committee. (C) ESPON, 2013. 
with densities up to 15,500 people $/ \mathrm{km}^{2}$, and sparsely populated rural areas with densities below 10 people $/ \mathrm{km}^{2}$.

Between 1998 and 2008, the population increased by 20\% to reach 14.2 million and the number of jobs grew by 2.1 million (50\%). In the case study area, the population is heavily concentrated along the coastal fringe of the Mediterranean Sea. The three main agglomerations are Barcelona (5 million population), Valencia (1.8 million) and Alacant-Elx (0.8 million).

Approximately $13 \%$ of the population are now foreigners, most of them people who came to fill job vacancies generated during the period of strong growth by 3.5\% GDP per year between 1997 and 2007, but a significant number were also attracted by the climate and the quality of life in the region. About one in five immigrants is from another EU state, and in some places the proportion stands at one in three, similar to the level in the American Sunbelt.

\section{Spatial structure}

The present territorial organisation of these regions involves a large number of jurisdictions and a complex distribution of responsibilities and resources between administrations. This introduces rigidities and inefficiencies in the public sector: on the one hand, it hinders achieving economies of scale in the supply of public services and, on the other, the difficulties of reaching consensus under very different political agendas often create insurmountable obstacles to carrying out major strategic projects.

\section{Socio-economic situation}

The West Mediterranean area has an important industrial tradition. The sector is characterised by the presence of some multinational companies attracted during the 1980s and 1990. Yet, it is essentially composed of a broad network of small and medium-sized family companies in mature sectors, sometimes constituting sectoral clusters in fields such as ceramics, footwear, textiles, toy industry, furniture, and agri-food. The relocation of some major multinational industrial companies may be inevitable; nonetheless, re-industrialisation of the territory is a major political objective in the mid-term.

With 60 million visitors per year, Spain ranks second only to France as the world's leading tourism destination. Some 30 million people visit the case study area every year (15 million visit Catalonia; 10 million - Balearic Islands; 5 million - Valencia). Tourism is expected to evolve towards the provision of additional services related to education and training, health and well-being, sport and leisure as well as cultural and business activities, and constitutes a strategic sector.

\section{Transport aspects}

The long distance road corridors of the case study area comprise essentially the Mediterranean axis along the coast (integrated by two parallel motorways), three axes linking the cost with Zaragoza (from Barcelona, Tarragona and Valencia) and two axes linking the coast with Madrid (from Valencia and Alacant). All these axes are served by motorways, and some of them are tolled. It is already difficult for the current road capacity in these corridors to meet the demand on many sections, notably around metropolitan areas. Particularly during peak tourism seasons, some motorway and trunk road sections reach congestion levels that are unacceptable. 


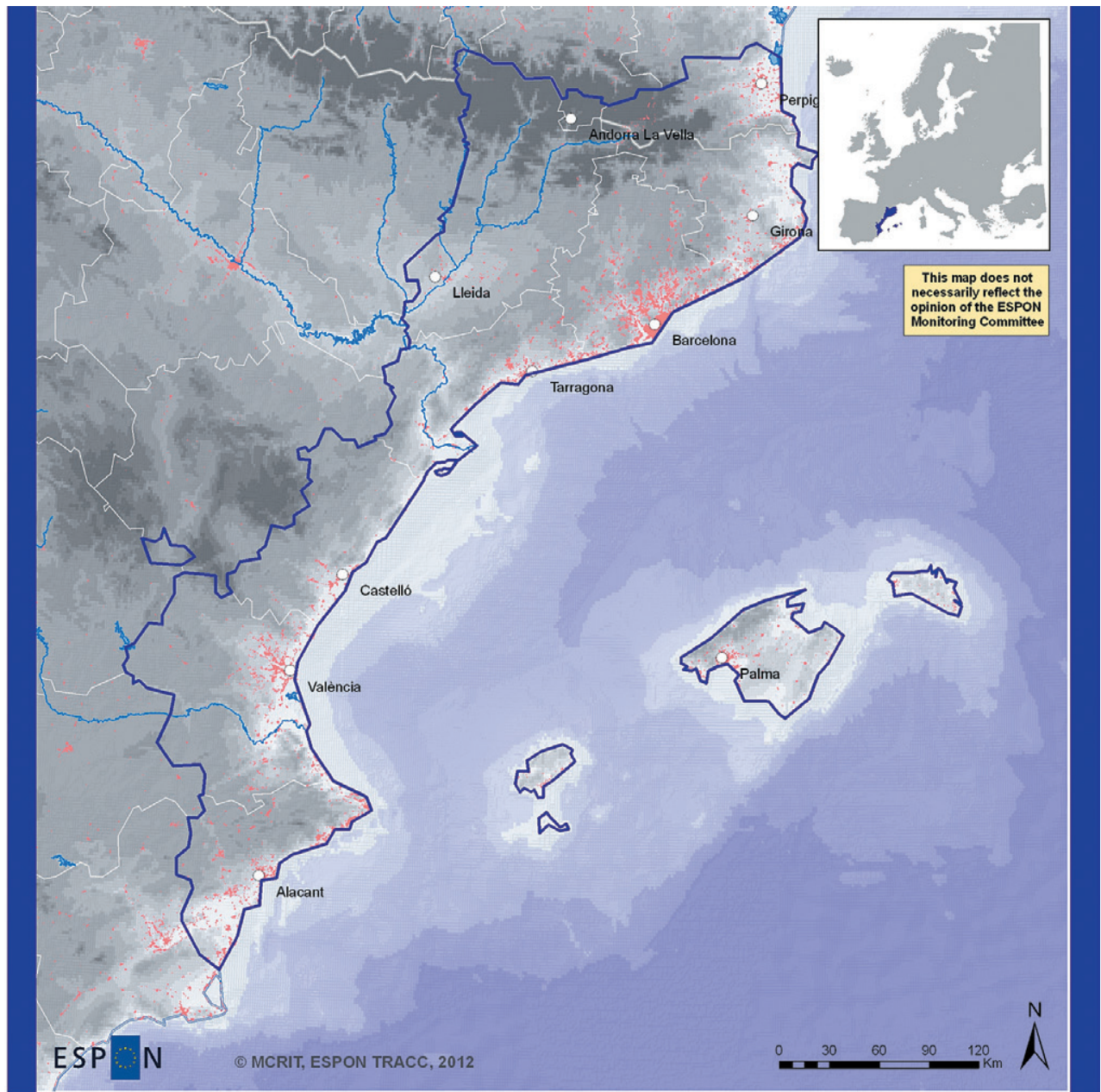

EUROPEAN UNION
Part-financed by the European Regional Development Fund
INVESTING IN YOUR FUTURE

Source: MCRIT, 2012

Origin of data: NOAA 2009; ESRI 2008; EEA 2006 (C) EuroGeographics Association for administrative boundaries

\section{West Mediterranean Regions}

Physical Framework

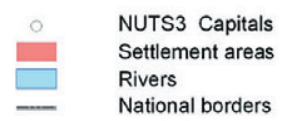

Figure 1. The West Mediterranean case study region.

Over a period of ten years, the Spanish high-speed train network has become one of the best developed in the world. There are many new sections being built across the Iberian Peninsula and, following the inauguration of the Barcelona-Madrid line in 2008 (6.2 million passengers in the corridor in 2010, 2.6 million between Barcelona and Madrid), the connection from Barcelona to France in Perpignan began only operating in 2013. The high speed railway line between Madrid and 
Valencia has been in service since December 2010 (2.4 million passengers in the corridor in 2011, 1.8 million between Valencia and Madrid). Along the coastline, Euromed rail services run between Alacant, Valencia, Castelló, Tarragona and Barcelona (2.5 million passengers in 2006) in an upgraded rail corridor that allows speed of $200 \mathrm{~km} / \mathrm{h}$, but is not yet integrated with the rest of the high speed network which runs on a different gauge.

\section{Accessibility patterns}

The accessibility model used to calculate travel times and costs for accessibility indicators is a network model developed by MCRIT in C++ on the BRIDGES platform. The model allows the assignment of Origin-Destination matrices at the LAU2 level of detail for both road and rail.

BRIDGES was developed within the Strategic Transport element of the 4th Framework Programme between 1997 and 1999. The main goal of developing BRIDGES software technology was to provide policy-makers, in particular those responsible for European transport policies, with friendly and productive access to advanced decision-making tools, such as transport models and harmonised databases. Research under BRIDGES was defined in the context of ideal user requirements for a European Transport policy Information System (ETIS) and the problems and opportunities presented by existing or expected software applications, data formats and transport strategic models needed to fulfil the user requirements of an ideal ETIS.

Transport supply is modelled by means of the road and rail networks. The key attribute of network links is speed. For the rail network, speed not only reflects the physical features of the link but also the commercial speed of services operating (i.e. speed takes into account the train timetable).

Each LAU2 zone is represented by a centroid connected to the road and, if available, also to the rail network. The route between each zone pair is determined using an assignment algorithm based on the shortest cost paths.

The model is used to estimate accessibility for car and for public transport, following the common methodology applied by the TRACC project (Biosca et al., 2013). In the latter, public bus is simulated by introducing reduced speeds on the road network leading towards railway stations. These reduced speeds take into account the average commercial speed of bus services in the West Mediterranean region, as well as average frequency for these services. Therefore, public road transport is not simulated based on actual timetables.

\section{Daily accessibility of jobs by car}

The West Mediterranean region has a labour market spreading over most of its territory, even if the density of activities is higher in coastal areas (as it is the case with the population), and especially high in the areas around Barcelona, Valencia and Alacant.

As a result of this level of density, around $65 \%$ of the population in the region has over 1 million jobs accessible in less than one hour by car, and more than $40 \%$ of the population has 2 million jobs accessible within 60 minutes' drive. Only a small part of the population, living in smaller areas located mainly in the mountains, can reach less than 100,000 jobs within 60 minutes. The area around the Ebre delta (between Tarragona and Castelló) remains an area of lower job availability compared to other parts of the region, despite its central position, due mostly to its sparse settlement structure and being too far from Barcelona and Valencia to reach their labour markets within a 60 minutes' drive. 
Logically, territories around the largest cities in the area display the highest values for job accessibility by public transport because of geographical proximity to the cities but, most importantly, because of having more public transport endowment, especially rail infrastructure and services. The number of jobs accessible in inland areas of the region is still relatively high, except for mountain areas in the western Pyrenees and in interior Valencia and Castelló.

\section{Regional accessibility potential by car}

The map of the accessibility potential by car to the population visualises the "enlarged cities" in the case study area, the metropolitan areas in a broad sense.

In Catalonia, the fairly concentric network of motorways around Barcelona becomes noticeable as accessibility potential decreases from the city into the backcountry following the major transport corridors. The influence of Barcelona is perceived in the four Catalan NUTS-3 capitals, in addition to Perpignan in France. In the south, the influence of Valencia also spreads widely into the territory, and almost merges with the metropolitan area of Alacant-Elx, even Murcia. The values for potential accessibility in the islands are limited.

Potential accessibility of jobs by public transport reflects the public transport corridors around the largest cities in the region. In Barcelona, the map reflects the trace of the suburban railway lines to Igualada, Manresa and Vic in the interior, and along the coast to Girona and Tarragona. The new high speed rail station in inner Tarragona province provides higher accessibility potential to jobs in the area, which cannot be seen in Girona yet as the high speed rail line had not been in service at the time of editing this paper. Suburban rail services can also be tracked in the Valencia metropolitan region, reaching up as far as Castelló del Plana.

\section{Access to regional centres by public transport}

Regional centres are defined as NUTS-3 capitals (centres of administrative services) or cities with a population of more than 50,000. In the West Mediterranean region, there are 42 regional centres distributed all over the study area, but particularly concentrated in coastal areas and around the largest metropolitan areas in Barcelona, Valencia and Alacant. In the neighbouring territories, there are around 10 additional regional centres, which are also taken into consideration in the analysis.

The accessibility of regional centres by car is relatively good for most of the LAU2 units. One out of five inhabitants of the West Mediterranean region (19\%) lives within 15 minutes' drive from a regional centre, half of the population lives within 30 minutes (52\%), and four out of five inhabitants (78\%) live within 45 minutes. More than $90 \%$ of the population live within 1 hour away from a regional centre. Municipalities with worse accessibility conditions are located in peripheral areas of the case study region, e.g. in the Pyrenees, in the backcountry mountains of Valencia, in the area around the Ebre delta, and on the smallest islands.

Travel time to regional centres by public transport is longer than by car. When taking access times, service frequencies, and commercial speeds into consideration, only $23 \%$ of inhabitants of the region live 30 minutes away from a regional centre by public transport, only $63 \%$ of the population live 60 minutes away and 83 minutes are needed for $80 \%$ of the total population to reach a regional centre. Low public transport accessibility is observed in mountain areas, but also in those parts of the study region where railway infrastructure is underdeveloped or simply inexistent (i.e. most of the interior areas). 
Access to health care facilities by car

In Spain, the public health care system has seen a very important development in the recent decades. Today, the majority of counties in the West Mediterranean region (NUTS-4) are served by a public hospital. For instance, in Catalonia there are at least 30 counties with a public reference public hospital (73\%), and in the Valencia and Balearic autonomous communities, the health care infrastructure endowment is also very high.

Accessibility of hospitals is in general better than accessibility of regional centres, reflecting that hospital endowment is often made available to municipalities under the threshold established by ESPON TRACC to consider regional centres ( $>50,000$ inhabitants and NUTS-3 capitals). $39 \%$ of population live 15 minutes away from a hospital (vs. 18\% for a regional centre), $81 \%$ of population live 30 minutes away (vs. 52\% for a regional centre), and $95 \%$ live 45 minutes away (vs. $78 \%$ for a regional centre). The territorial pattern of the accessibility of hospitals is more homogeneous than of regional centres, and good performance spreads in most of the territory under analysis.

The pattern of public transport is similar to that of private transport, but the influence of hospitals in the territory is more limited, especially in the most peripheral regions. The examples are: Requena -in the backcountry of Valencia - or Vielha and Tremp in the Catalan Pyrenees. In the West Mediterranean region, 16\% of population live 15 minutes away from a hospital by public transport, $47 \%$ live 30 minutes away, $74 \%$ live 45 minutes away, and $88 \%$ of the population covered by the case study live 1 hour away from a hospital. 


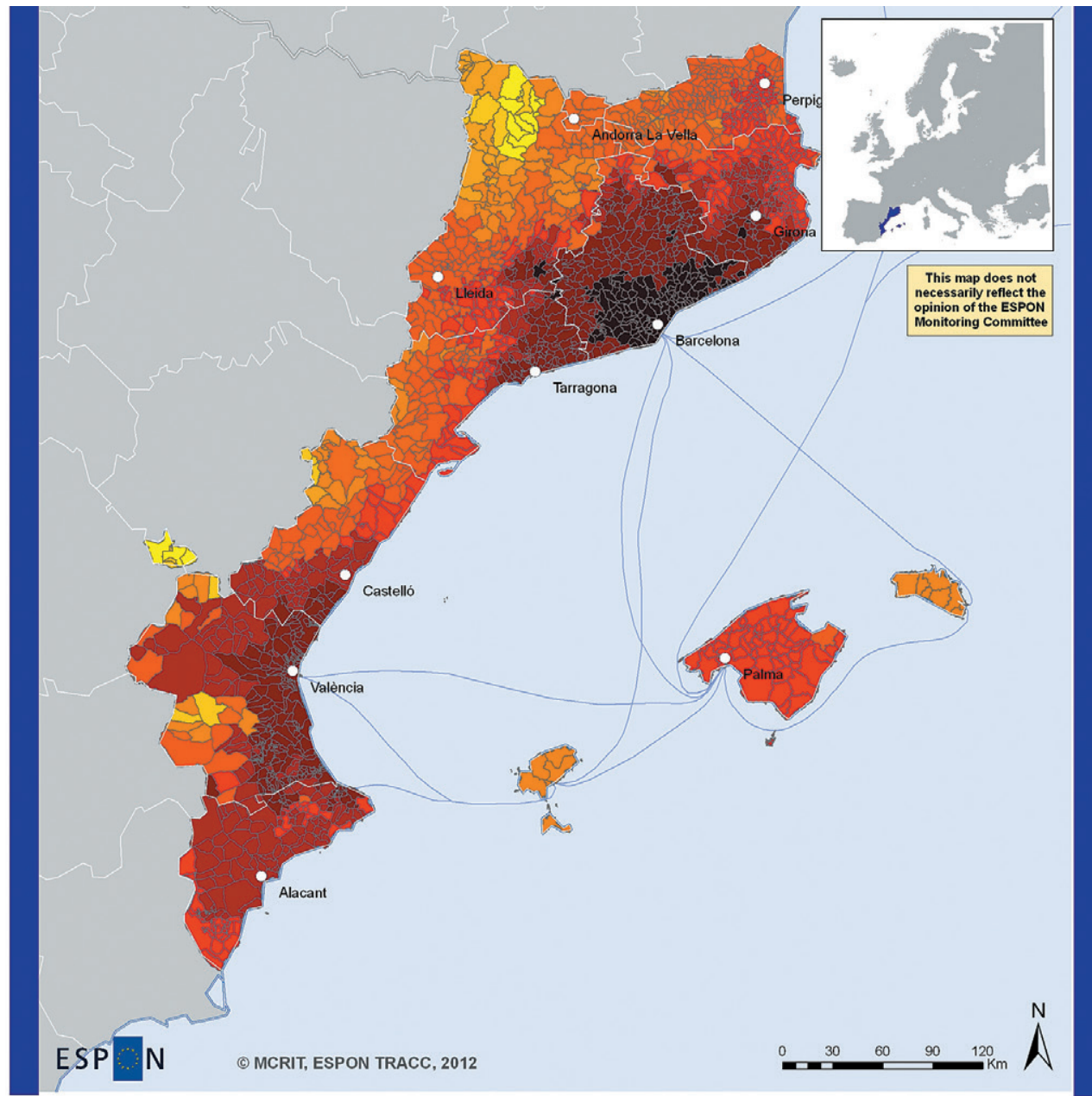

EUROPEAN UNION

EUROPEANUNION European Regional Development Fund
Part financed b the EUTESTING IN YOUR FUTURE

Source:MCRIT, 2012 Origin of data: INSS (Spain), INSEE (France), Statistics Department of Andorra, 2011 (1) Euro Geographics Association for administrative boundaries

West Mediterranean Regions

Daily accessibility of jobs by car

\begin{tabular}{|c|c|c|c|}
\hline $0-2.500$ & $100.001-250.000$ & 0 & NUTS3 \\
\hline $2.501-5.000$ & $250.001-500.000$ & & \\
\hline $5.001-10.000$ & $500.001-1.000 .000$ & & \\
\hline $10.001-50.000$ & $1.000 .001-2.500 .000$ & & \\
\hline $50.001-100.000$ & $2.500 .000<$ & & \\
\hline
\end{tabular}

Figure 2. Jobs accessible within 60 minutes by car. 


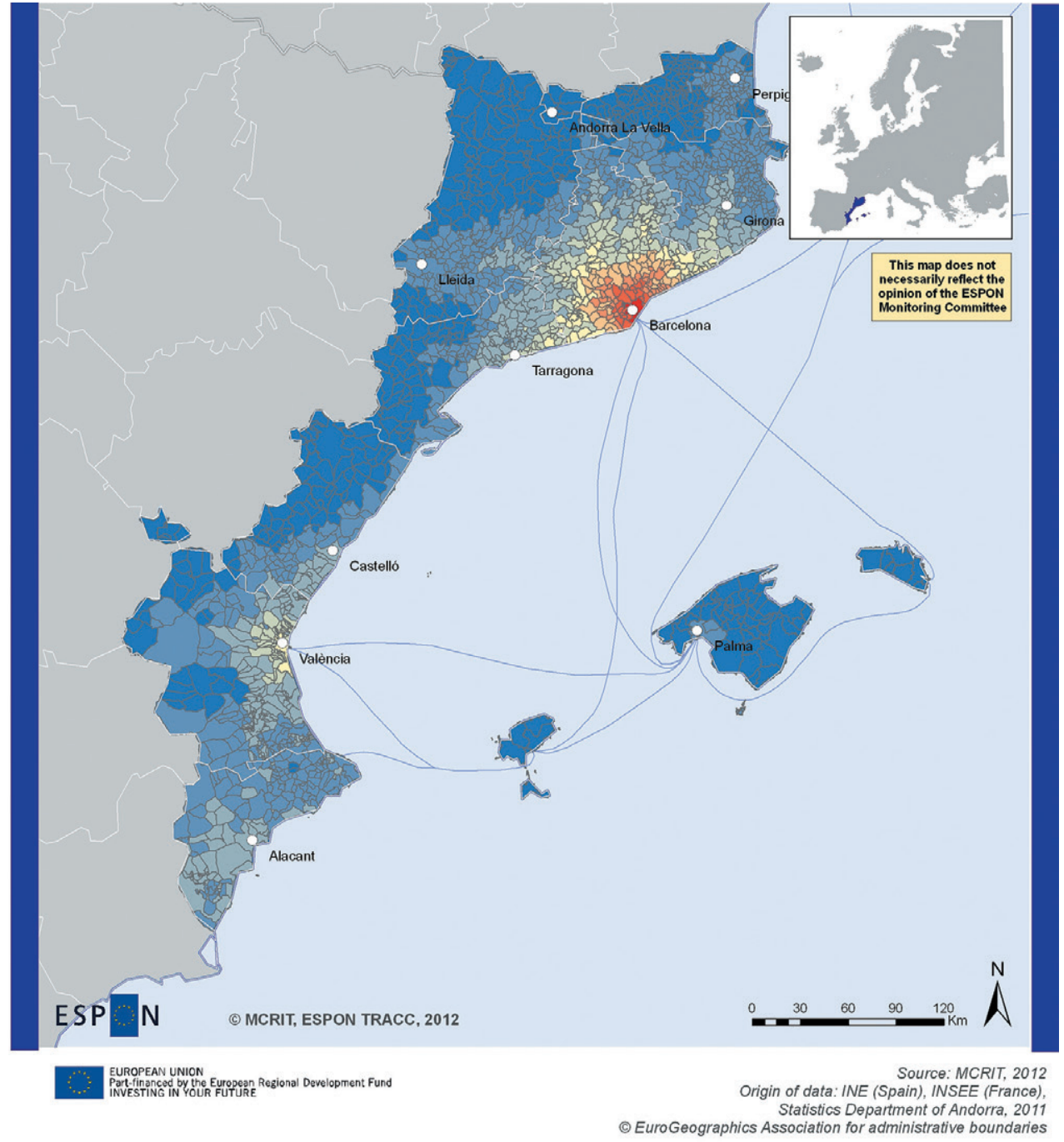

\section{West Mediterranean Regions}

Potential accessibility to population by car.

Regional average $=\mathbf{1 0 0}$
$0,0-25,0$
$25,1-50,0$
$50,1-75,0$
$75,1-100,0$
$100,1-125,0$

\begin{tabular}{|l|l}
\hline & $125,1-150,0$ \\
$\square$ & $150,1-175,0$ \\
& $175,1-200,0$ \\
$\square$ & $200<$ \\
\hline$\quad$ NUTS 3 Capital
\end{tabular}

Figure 3. Potential accessibility to population by car. 


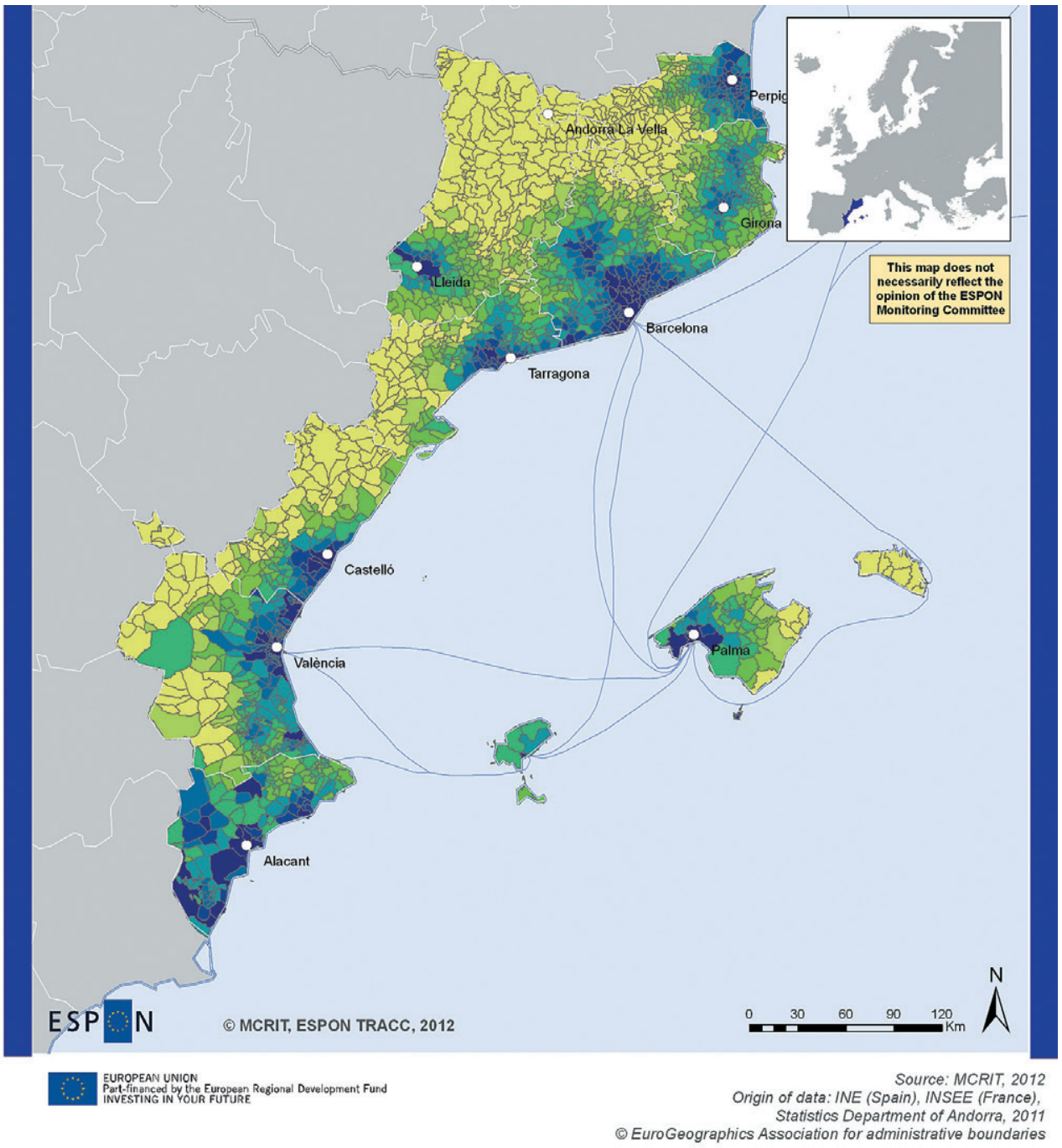

West Mediterranean Regions

Travel time to regional centre by public transport ( $\mathrm{min})$

$0-10$

$51-60$

$11-20$

$21-30$

$31-40$

$41-50$

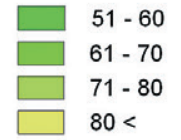

NUTS 3 Capital

Figure 4. Travel time to nearest regional centre by public transport. 


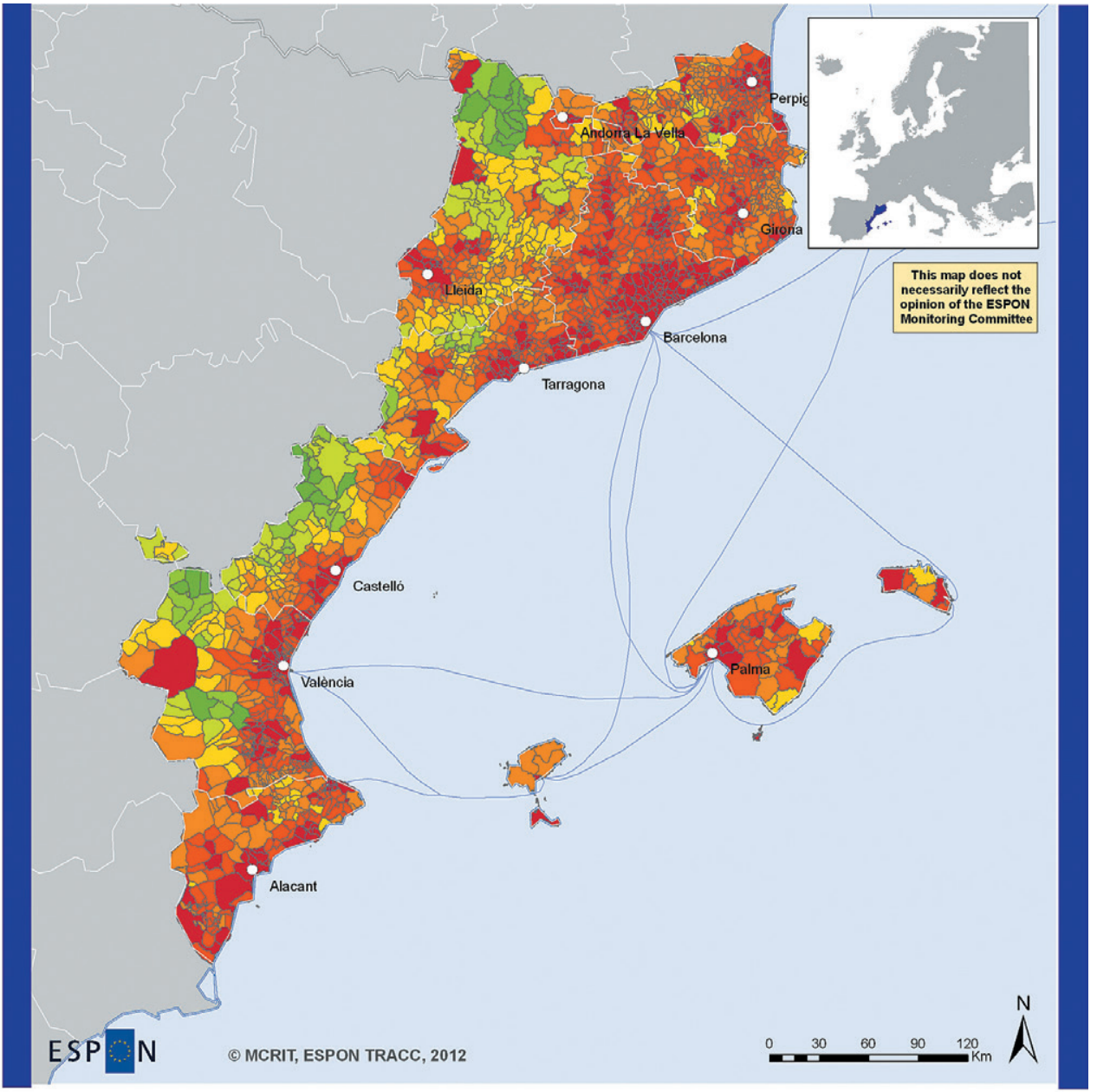

EUROPEAN UNION
Part-financed by the Europegn Regional Davelopment Fund
INVESTING IN YOUR FUTURE

Origin of data: Hospitals database: Ministerio de Sanidad (Spain), anté du Languedoc-Roussillon (France), Govern d'Andorra, 2011 (c) EuroGeographics Association for administrative boundaries

\section{West Mediterranean Regions}

Travel time to closest hospital by car ( $\mathrm{min})$
$0-10$
$41-50$
$11-20$
$51-60$
$21-30$
$60<$
$31-40$
NUTS 3 Capital

Figure 5. Travel time to closest hospital by car. 


\section{The impact of the future TEN-T network developments}

The long distance road corridors of the West Mediterranean region comprise essentially the Mediterranean axis all along the coast (constituted by two parallel motorways: AP-7 // A-7), the Eix de l'Ebre linking Madrid and Zaragoza with Barcelona (constituted by the AP-2 // A-2 motorways), the Valencia - Zaragoza corridor (A-23) and the route from Valencia to Madrid (A-3 // A-31).

All these corridors belong to the TEN-T road network, some to the core network and others to the comprehensive network. Additionally, it is planned that the TEN-T comprehensive network would upgrade three trans-Pyrenees international crossings linking Toulouse with Barcelona, Lleida and Zaragoza (from the east to the west). The West Mediterranean case study has integrated all planned upgradings on any TEN-T road corridor in the region to constitute the future scenario to be tested.

There are many new railway sections being built across the Iberian Peninsula and, in particular, in the West Mediterranean region. Most of the projects have been slowed down by the economic crisis, and some may only be constructed over a very long period of time. All high speed railway lines in Spain are included in the TEN-T rail network.

Following the inauguration of the Barcelona-Madrid line in 2008, and the Valencia-Madrid line in 2010, the high speed rail link from Barcelona to Perpignan became only fully operational by late 2013, and the stretch from Perpignan to Montpellier necessary to connect the Spanish network with the rest of Europe will most likely be upgraded in the medium term. In the Autonomous Community of Valencia, the three provincial capitals (NUTS-3 capitals) are planned to be interlinked and also connected with Madrid in the medium term, but projects are uncertain now due to the economic crisis. The corridor from Valencia to Zaragoza is also to be upgraded to high speed standards in the future. In the short term, the continuity of the high-speed Mediterranean corridor between Valencia and Barcelona requires upgrading a $30 \mathrm{~km}$ link right south of Tarragona, which has already been under construction before the crisis, but works stopped now due to funding shortages. This stretch should allow trains to reduce present travel time by some 45 minutes between Barcelona and Valencia (from $3 \mathrm{~h}$ to $2.15 \mathrm{~h}$ ). The West Mediterranean case study has integrated all planned upgradings on any TEN-T rail corridor in the region to constitute the future scenario to be tested.

Impacts of the TEN-T are especially important in rail. Next figure shows the trace of the future high speed rail lines in the new potential accessibility pattern. High values of accessibility to population are recorded in all the cities having stations of the train: from north to south, Perpignan, Figueres, Girona, Barcelona, Tarragona, Tortosa, Castelló, Valencia and Alacant, and in the interior, Lleida. The largest void remains located between Tortosa in the southern edge of Catalonia, and Cas-telló in northern autonomous community of Valencia, which being a sparsely populated area has no high speed rail stations planned. 


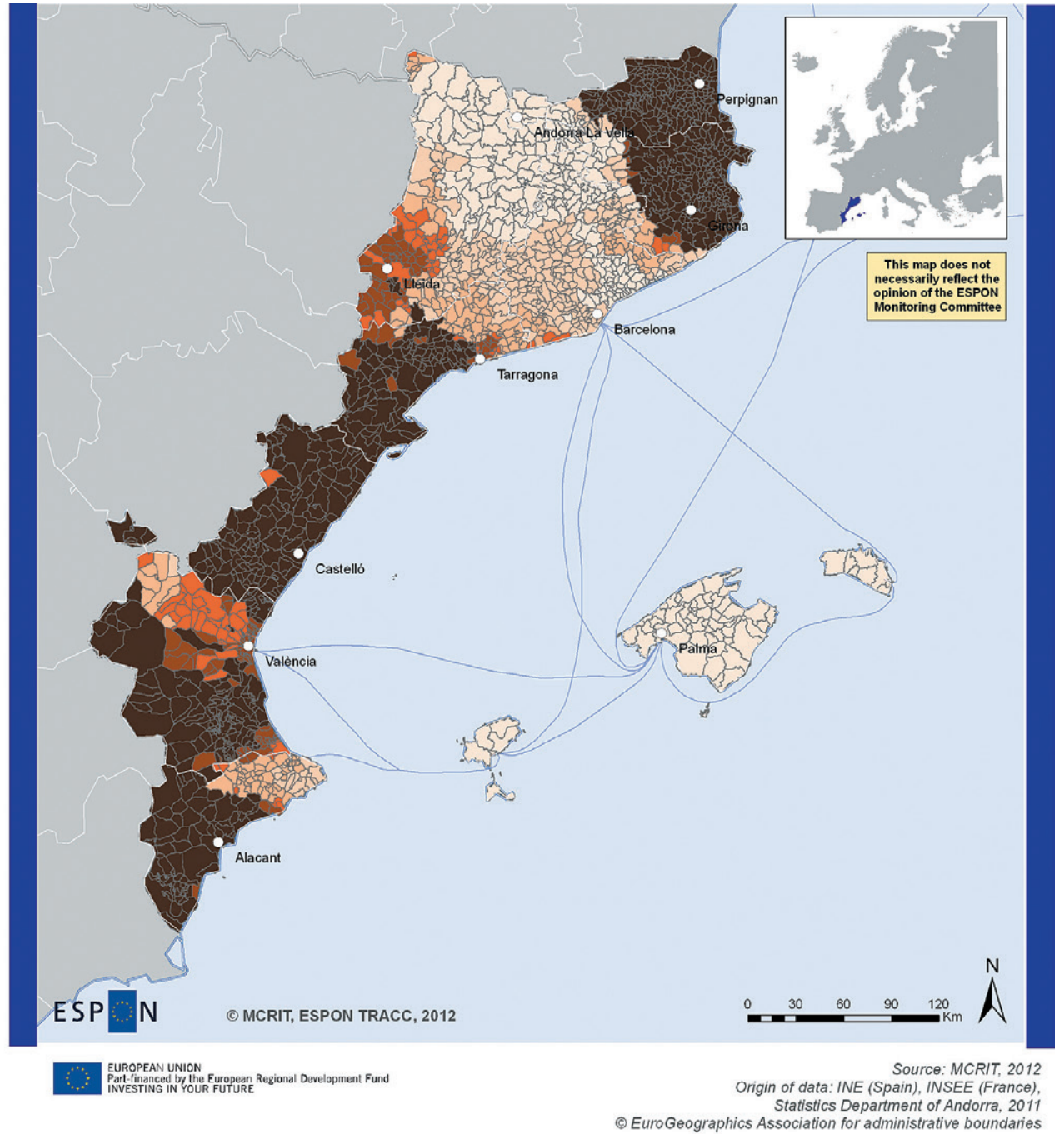

West Mediterranean Regions

Potential accessibility to population by car (relative change, \%)

(standardised on public transport average 2010)

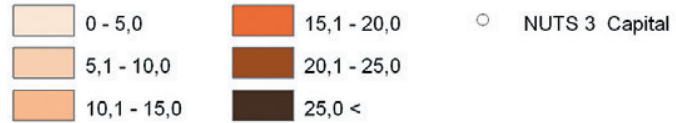

Figure 6. Relative increase in potential accessibility to population by car with TEN-T projects. 


\section{Conclusions}

In the West Mediterranean region, the accessibility patterns follow approximately the geographic constraints of the region which, being a narrow coastal corridor for most of the territory, concentrates most of the population and activities at the seaside. The backcountry is substantially less populated, especially in mountain areas; infrastructure endowment is more limited giving place to poorer accessibility patterns in comparison.

In terms of accessibility, the region is dominated by two big metropolitan agglomerations of Barcelona and Valencia. Most of the maps show that the influence of Barcelona agglomeration at large reaches approximately all NUTS-3 capitals in Catalonia, and in some cases even Perpignan in France. In the south, the metropolitan agglomerations of Valencia and Alacant tend to merge with each other in most of the cases, despite the fact that abrupt topography changes in the areas in between both agglomerations provides locally some areas of low accessibility (e.g. inner peripheries in Valencian Comarques Centrals, Alcoi).

There is a clear difference in terms of accessibility between private car and public transport, the latter performing worse than the former, as expected. In this regard, the impact of the new high speed rail lines all over the region (planned lines are all included in the rail TEN-T) and increasing the opportunities of medium-sized cities in between the largest agglomerations are especially relevant. These cities may be in a position to offer better living standards and allow everyday commuting to the main labour markets, but may also be threatened to become mere residential economies due to rising competition from more robust markets.

All over the region, accessibility of public services is more homogenous than accessibility of the population and jobs, reflecting the fact that public services are relatively decentralised in the Western Mediterranean regions, with the ratios of hospitals or schools per 1,000 inhabitants higher in areas with relatively low populations than in the largest agglomerations.

The case of the Balearic Islands shows the impact of insularity on accessibility patterns. Each island remains an isolated region, despite the existence of fast ferries linking some of the islands with each other and frequent internal flights.

\section{References}

Biosca O., Spiekermann K., Stępniak M., 2013. Transport accessibility at regional scale, EUROPA XXI, vol. 24, pp. 5-17.

MCRIT, 2011. Modelling module for interconnectivity, Deliverable D5.3 of INTERCONNECT, Co-funded by FP7. TRI, Edinburgh: Edinburgh Napier University.

MCRIT, Keys to bring advanced transport models to light. SPOTLIGHTS TN, Co-funded by FP5. MCRIT 2001-2002.

MCRIT, 1999. Implementation of ICON-GIS to the European Investment Bank.

MCRIT, A new middleware technology. BRIDGES, Co-funded by FP4. MCRIT 1998-2000. 
http://rcin.org.pl 